P. Muldowney, School of International Business, Magee College, University of Ulster, Londonderry, BT48 7JL, Northern Ireland. e-mail:

p.muldowney@ulst.ac.uk

\title{
THE HENSTOCK INTEGRAL AND THE BLACK-SCHOLES THEORY OF DERIVATIVE ASSET PRICING
}

\begin{abstract}
The classical Black-Scholes-Merton method for pricing European call options uses the Itô calculus to model the processes involved. We show how to model stochastic process using Henstock integrands instead of Itô differentials (or stochastic integrals), and we show how to derive the Black-Scholes partial differential equation and pricing formulae using elementary methods.
\end{abstract}

\section{Introduction}

The Black-Scholes model [1] assumes that the price of an economic asset, as a random function of time, is a geometric Brownian motion. This implies that if the value $x_{j-1}$ occurs at time $t_{j-1}$, the probability of the outcome that, at time $t_{j}$ the process takes a value $x_{j}$ between $u_{j}$ and $v_{j}$, is related to

$$
\int_{u_{j}}^{v_{j}} \frac{1}{A} \frac{1}{x_{j}} \exp \left[-\frac{\left(\ln x_{j}-\ln x_{j-1}\right)^{2}}{2 \sigma^{2}\left(t_{j}-t_{j-1}\right)}\right] d x_{j}
$$

where $A$ is a normalizing factor $\left(2 \pi \sigma^{2}\left(t_{j}-t_{j-1}\right)\right)^{\frac{1}{2}}$.

When pricing a derivative asset, such as a European call option (see [2], and (4) below), whose value depends on the movements in the value of an underlying asset, the probabilities involved turn out to have the form

$\int_{u_{j}}^{v_{j}} \frac{1}{A} \frac{1}{x_{j}} \exp \left[-\left(\frac{\left\{\ln x_{j}-\ln x_{j-1}-\left(r-\frac{1}{2} \sigma^{2}\right)\left(t_{j}-t_{j-1}\right)\right\}^{2}}{2 \sigma^{2}\left(t_{j}-t_{j-1}\right)}\right)\left(t_{j}-t_{j-1}\right)\right] d x_{j}$,

Key Words: Henstock integral, Black-Scholes theory, derivative pricing

Mathematical Reviews subject classification: 26A39, 90A09, 90A12

Received by the editors March 8, 1999 
where $r$ is the (always positive) risk-free interest rate.

From this, the probability of the outcome that, at times $t_{j}$, the underlying asset price process $x$ takes values $x_{j}$ in the range $\left[u_{j}, v_{j}\right.$ [ for $1 \leq j \leq n$ will be given by integrating this from $u_{j}$ to $v_{j}, j=1,2, \ldots, n$, giving an integral of the form

$$
\int_{u_{1}}^{v_{1}} \cdots \int_{u_{n}}^{v_{n}}\{\cdots \exp [\cdots]\} d x_{1} \cdots d x_{n} .
$$

The pricing theory that has developed over the past twenty five years or so ([4], [5]) requires that the simple sets of outcomes described above be extended, using the Kolmogorov Theorem, to a sigma-algebra of measurable sets in an infinite-dimensional sample space whose representative elements are continuous paths, that the processes involved be represented by appropriate stochastic differential equations, that a suitable measure for the sample space be found by means of the Girsanov and Radon-Nikodym Theorems, and that the derivative asset valuation be then determined by means of an expectation using Lebesgue integration.

However, it is well known (see, for instance, [2]) that the so-called discrete time model of derivative valuation, in which only a finite number of times $t_{j}$ are considered, is much simpler than the continuous time model in which every possible time $t$ is allowed.

If we take, for instance, a European call option, whose dependence on the underlying asset value has a very simple form, and if we obtain a statistical expectation by integrating this, in $n$ dimensions only, with respect to the probabilities defined by the $n$-dimensional integrals above, we get a result similar to that which is obtained by the Lebesgue integral-based continuoustime model. See, for example, [2], page 91, and (4) below.

Furthermore, if take the integrand involved in this expectation and perform various partial differentiations on it, we obtain an equation similar to the classical Black-Scholes partial differential equation (see equation (5) below, also [2], page 95$)$.

These simplistic observations suggest there may be easier ways to formulate a continuous time model than those currently in use, and this is what motivates the following analysis which is guided by the methods and concepts of Henstock integration. The naive arguments described above are developed in this paper and the results are a generalization of the Lebesgue integral-based continuoustime Black-Scholes model. 


\section{Preliminaries}

Let $\mathbb{R}_{+}$denote the positive real numbers, and let $\overline{\mathbb{R}}_{+}$denote the positive real numbers with 0 and $+\infty$ adjoined. Let $x(t)$ be zero, or a positive real number, or $+\infty$ for each $\left.t \in] t_{0}, T\right]$, and let $x$ denote an element of $\overline{\mathbb{R}}_{+}^{\left.] t_{0}, T\right]}$. Let $\left.\left.t_{j} \in\right] t_{0}, T\right]$ for $j=1, \ldots, n$. Let $N$ denote a finite set of dimensions $\left\{t_{1}, \ldots, t_{n}\right\}$. It is generally convenient to include $T$ in $N$, so $t_{n}=T$. Let $I_{j}=I\left(t_{j}\right)$ denote an interval, in the $t_{j}$ dimension of $\mathbb{R}_{+}^{\left.\mid t_{0}, T\right]}$, of the form $] 0, v_{j}\left[\right.$ or $\left[u_{j}, v_{j}\left[\right.\right.$ or $\left[u_{j}, \infty[\right.$, where $u_{j}$ and $v_{j}$ are finite real numbers. Let $\left|I_{j}\right|$ denote 0 , or $v_{j}-u_{j}$, or 0 , respectively. Given $x(t)$ and $I(t)$, respectively a point of $\overline{\mathbb{R}}_{+}$and an interval of $\mathbb{R}_{+}$, we say that $x(t)$ and $I(t)$ are attached (or associated) if, respectively,

$$
\begin{aligned}
& x(t)=0 \quad \text { and } \quad I(t)=] 0, v[, \quad \text { or } \\
& x(t)=u \text { or } v \quad \text { and } \quad I(t)=[u, v[, \quad \text { or } \\
& x(t)=+\infty \quad \text { and } \quad I(t)=[u, \infty[.
\end{aligned}
$$

Let $I(N)$ denote $I_{1} \times \cdots \times I_{n}$, and let $|I(N)|$ denote $\prod_{j=1}^{n}\left|I_{j}\right|$. Given $x(N) \in$ $\overline{\mathbb{R}}_{+}^{N}$ and $I(N)$ an interval of $\mathbf{R}_{+}^{N}$, we say that $x(N)$ and $I(N)$ are attached (or associated) if $x_{j}$ and $I_{j}$ are attached for each $t_{j} \in N$. Let $I=I[N]$ denote

$$
\left\{x \in \mathbb{R}_{+}^{]_{0}, T\right]}: x_{j} \in I_{j}, t_{j} \in N\right\}
$$

so $I$ or $I[N]$ is the product of the finite-dimensional $I(N)$ times uncountably many copies of $\mathbb{R}_{+}$, one copy for each $\left.\left.t \in\right] t_{0}, T\right] \backslash N$. Let $|I[N]|:=|I(N)|$. Given $x \in \overline{\mathbb{R}}_{+}^{\left.\mid t_{0}, T\right]}$ and $I[N]$ an interval of $\mathbb{R}_{+}^{\left[t_{0}, T\right]}$ we say that $x, N$ and $I[N]$ are attached (or associated) if $x(N)$ and $I(N)$ are attached in $\mathbb{R}_{+}^{N}$.

This completes the basic definitions needed for a sample space with geometric Brownian motion. And the Henstock integral needed to compute statistical expectation of random variables in the space can now be defined by the method described in [8]. Some problems of alternative definitions of such integrals are discussed in a forthcoming article to be entitled, "Equivalent forms of the Black-Scholes integrand". This deals especially with correct ways of formulating Henstock gauges for Riemann sums in infinite dimensional domains.

\section{Geometric Brownian Motion}

Let $w$ denote an observable (or random variable) which is normally distributed with mean $\mu$ and standard deviation $\sigma$. Then $z=\exp w$ is said to be lognor- 
mally distributed, with probability density function

$$
f(z)=\frac{1}{\sqrt{2 \pi}} \frac{1}{\sigma z} \exp \left(-\frac{(\ln z-\mu)^{2}}{2 \sigma^{2}}\right),
$$

variance $e^{2 \mu+\sigma^{2}}\left(e^{\sigma^{2}}-1\right)$, and mean value (or expectation)

$$
E(z):=\int_{0}^{\infty} z f(z) d z=\exp \left(\mu+\frac{1}{2} \sigma^{2}\right) .
$$

For details of the evaluation of these integrals, see, for example, [6], pages 23 to 24 .

Let the process $x(t), t_{0}<t \leq T$, be a geometric Brownian motion (or, in other words, an exponential Wiener process). This means that $y(t)=\ln x(t)$ is a Brownian motion (or Wiener process), [8].

So if $y_{j-1}$ is determined, then $y_{j}-y_{j-1}$ is normally distributed with mean value $\mu\left(t_{j}\right)\left(t_{j}-t_{j-1}\right)$ and variance $\sigma^{2}\left(t_{j}-t_{j-1}\right)$. The Brownian motion $y(t)$ is said to have drift rate $\mu(t)$ and variance rate $\sigma^{2}$. In the usual notation for conditional expectation, $y_{j}$ is normally distributed with mean $y_{j-1}+\mu\left(t_{j}\right)\left(t_{j}-\right.$ $\left.t_{j-1}\right)$;

$$
E\left(y_{j}-y_{j-1} \mid y_{j-1}\right)=\mu\left(t_{j}\right)\left(t_{j}-t_{j-1}\right), \quad E\left(y_{j} \mid y_{j-1}\right)=y_{j-1}+\mu\left(t_{j}\right)\left(t_{j}-t_{j-1}\right) .
$$

By these properties of Brownian motion, and by the remarks concerning the lognormal distribution above,

$$
E\left(\frac{x_{j}}{x_{j-1}} \mid x_{j-1}\right)=\exp \left[\mu\left(t_{j}\right)\left(t_{j}-t_{j-1}\right)+\frac{1}{2} \sigma^{2}\left(t_{j}-t_{j-1}\right)\right] .
$$

Therefore if $y$ has a drift rate of $\mu(t)-\frac{1}{2} \sigma^{2}$, then

$E\left(\frac{x_{j}}{x_{j-1}} \mid x_{j-1}\right)=\exp \left(\mu\left(t_{j}\right)\left(t_{j}-t_{j-1}\right)\right)$, or $E\left(x_{j} \mid x_{j-1}\right)=x_{j-1} e^{\mu\left(t_{j}\right)\left(t_{j}-t_{j-1}\right)}$,

and we say that $x$ has a growth rate of $\mu(t)$.

If $\sigma^{2}$ is the variance rate of the Brownian motion $y=\ln x$, then $\sigma$ is the volatility of the geometric Brownian motion $x$. So if the Brownian motion $y=\ln x$ has drift rate $\mu$, then the geometric Brownian motion $x=e^{y}$ has growth rate $\mu+\frac{1}{2} \sigma^{2}$.

As we can see, all of these features of geometric Brownian motion are derived from elementary properties of the normal and lognormal probability density functions. Throughout the remainder of this paper, whenever we 
invoke the properties of Brownian motion or of geometric Brownian motion (including those cases where the probability is taken to be concentrated on the subset of $x \in \mathbb{R}^{\tau \tau, T]}$ which are continuous) we assume $\sigma(t)$ to be constant for all $t$. In a future publication it will be shown how this condition may be relaxed.

With $\sigma(t) \in \mathbb{R}_{+}$defined for $\left.\left.t \in\right] t_{0}, T\right]$, let $\sigma_{j}$ denote $\sigma\left(t_{j}\right)$. Consider the following expression.

$$
\prod_{j=1}^{n}\left[\exp \left\{-\frac{1}{2 \sigma_{j}^{2}} \frac{\left(\ln x_{j}-\ln x_{j-1}\right)^{2}}{t_{j}-t_{j-1}}\right\}\right]\left[2 \pi \sigma_{j}^{2}\left(t_{j}-t_{j-1}\right)\right]^{-\frac{1}{2}} .
$$

Denote this expression by $g(x(N))$. For a given $N$, this notation implies $g(x(N))$ is a function defined in a finite-dimensional space $\mathbb{R}_{+}^{N}$. Let

$$
g(x(N), I(N)):=g(x(N)) \prod_{j=1}^{n} \frac{\left|I_{j}\right|}{x_{j}}, \quad G(I(N)):=\int_{I(N)} g(x(N), J(N)) .
$$

The integral is a finite-dimensional Henstock integral found by taking Riemann sums $(\mathcal{E}) \sum g(x(N), J(N))$ in which $I(N)$ is partitioned by sub-intervals $J(N)$. So if $I(N)=\prod_{j=1}^{n}\left[u_{j}, v_{j}[\right.$, then $G(I(N))$ is

$\int_{u_{1}}^{v_{1}} \cdots \int_{u_{n}}^{v_{n}} \prod_{j=1}^{n}\left[\exp \left\{-\frac{\left(\ln x_{j}-\ln x_{j-1}\right)^{2}}{2 \sigma_{j}^{2}\left(t_{j}-t_{j-1}\right)}\right\}\right]\left[2 \pi \sigma_{j}^{2}\left(t_{j}-t_{j-1}\right)\right]^{-\frac{1}{2}} \frac{d x_{1}}{x_{1}} \cdots \frac{d x_{n}}{x_{n}}$.

In the infinite-dimensional space $\mathbb{R}_{+}^{\left[t_{0}, T\right]}$, if $x, N, I[N]$ are associated, let

$$
g(x, N, I):=g(x(N), I(N)), G(I[N]):=G(I(N)) .
$$

Sometimes we wish to integrate $G(I[N])$ in $\mathbb{R}_{+}^{\left.] t_{0}, T\right]}$. Then we require the integrand to be defined for each associated $x, N, I$. In this case we may take

$$
G(x, N, I):=G(I[N]) .
$$

\section{Properties of Geometric Brownian Motion}

Much of the following flows from the theory of Brownian motion, expressed in terms of Henstock integration instead of Lebesgue integration, as it has been presented in [7] and [8], but with $\ln x(t)$ in place of $x(t)$, and with $\mathbb{R}_{+}^{\left.] t_{0}, T\right]}$ in place of $\mathbb{R}^{\left.] t_{0}, T\right]}$. 
Proposition 1. $g(x, N, I)$ is variationally equivalent to $G(x, N, I)$.

Proof. This follows from [7], page 54, Proposition 37, if we replace $x(t)$ by $e^{x(t)}$ in (1) above.

As in the construction of Brownian motion (see [8] and [9]), we can define a so-called "continuous modification" of $G$ so that, in effect, $\mathbb{R}_{+}^{\left[t_{0}, T\right]}$ can be replaced as the sample space by the subset $C$ of continuous $x$ in $\mathbb{R}_{+}^{\left[t_{0}, T\right]}$. (If $x \in \overline{\mathbb{R}}_{+}^{\left.t_{0}, T\right]} \backslash \mathbb{R}_{+}^{\left.t_{0}, T\right]}$, then $x \notin C$.) To do this, use the arguments of [7], Theorem 5 , and [9], Section 6 , but replacing $x(t)$ by $\ln x(t)$ in those arguments. If we denote the continuous modification of $G$ by $Q$, then

$$
Q(x, N, I)=\left\{\begin{array}{lll}
G(I[N]) & \text { if } & x \in C, \\
0 & \text { if } & x \in \overline{\mathbb{R}}_{+}^{\left.t_{0}, T\right]} \backslash C .
\end{array}\right.
$$

In this case we make use of the fact that

$$
\int_{\mathbb{R}_{+}^{] t_{0}, T\right]}} \mathbf{1}_{C_{N} \cap I[N]}(x) G(J[M])=G(I[N]),
$$

which follows from [7] page 52, Proposition 36, and page 61, Proposition 46, making the appropriate change of variable. $C_{N}$ is the set of $x$ which are continuous at each $t \in N$. (In the integral, in effect the infinite-dimensional cylindrical interval $I[N]$ is partitioned by infinite-dimensional cylindrical intervals $J[M]$ with $M \supseteq N$.)

$Q(x, N, I)$ is the probability that the sample path $x$ of a geometric Brownian motion satisfies $x(t) \in I(t)$ for $t \in N$.

Let $\left.\left.M=\left\{\tau_{1}, \ldots, \tau_{m}\right\} \subset\right] t_{0}, T\right]$ be fixed and suppose a functional $h$ satisfies $h(x)=h(x(M))$ for all $x$. Then $h$ is called a cylinder functional. $h$ depends only on the values taken by $x$ at $\tau_{1}, \ldots, \tau_{m}$, and we can treat it as a function of $x(M) \in \mathbb{R}^{M}$ or as a function of $x \in \mathbb{R}_{+}^{\left.] t_{0}, T\right]}$.

Proposition 2. If $h$, considered as a function of $x(M) \in \mathbb{R}^{M}$, is almost everywhere continuous, and if $h(x(M)) G(I(M))$ is Henstock integrable in $\mathbb{R}^{M}$, then $h(x) G(I[N])$ is Henstock integrable in $\mathbb{R}_{+}^{\left.\mid t_{0}, T\right]}$, and

$$
\int_{\mathbb{R}_{+}^{\left.t_{0}, T\right]}} h(x) G(I[N])=\int_{\mathbb{R}^{M}} h(x(M)) G(I(M)) .
$$

Proof. Follows from [7], page 56, Proposition 38, replacing $x(t)$ by $\ln x(t)$. 
Let $\mu(t)$ be real-valued for $\left.t \in] t_{0}, T\right]$, and write $\mu_{j}$ for $\mu\left(t_{j}\right)$. Consider the expressions

$$
\begin{gathered}
\prod_{j=1}^{n} \frac{\left[\exp \left\{-\frac{1}{2 \sigma_{j}^{2}}\left(\frac{\ln x_{j}-\left\{\ln x_{j-1}+\left(\mu_{j}-\frac{1}{2} \sigma_{j}^{2}\right)\left(t_{j}-t_{j-1}\right)\right\}}{t_{j}-t_{j-1}}\right)^{2}\left(t_{j}-t_{j-1}\right)\right\}\right]}{\left[2 \pi \sigma_{j}^{2}\left(t_{j}-t_{j-1}\right)\right]^{\frac{1}{2}}}, \\
\prod_{j=1}^{n} \exp \left\{\frac{1}{\sigma_{j}^{2}}\left(\mu_{j}-\frac{1}{2} \sigma_{j}^{2}\right)\left(\ln x_{j}-\ln x_{j-1}\right)-\frac{1}{2 \sigma_{j}^{2}}\left(\mu_{j}-\frac{1}{2} \sigma_{j}^{2}\right)^{2}\left(t_{j}-t_{j-1}\right)\right\} .
\end{gathered}
$$

Denote (3) by $V(x, N ; \mu, \sigma)$, which we will write as $V, V(x, N)$ or $V(\mu, \sigma)$, as and when it suits us; and observe that (2) is the same as (1) multiplied by $V(x, N ; \mu, \sigma)$. Denote $(2)$ by $g_{\mu \sigma}(x(N))$. Thus

$$
g_{\mu \sigma}(x(N))=g(x(N)) V(x, N ; \mu, \sigma) .
$$

Let

$$
\begin{aligned}
g_{\mu \sigma}(x(N), I(N)) & :=g_{\mu \sigma}(x(N)) \prod_{j=1}^{n} \frac{\left|I_{j}\right|}{x_{j}}, \\
g_{\mu \sigma}(x, N, I) & :=g_{\mu \sigma}(x(N), I(N)), \\
G_{\mu \sigma}(I(N)) & :=\int_{I(N)} g_{\mu \sigma}(x(N), J(N)), \\
G_{\mu \sigma}(I[N]) & :=G_{\mu \sigma}(I(N)), \\
G_{\mu \sigma}(x, N, I) & :=G_{\mu \sigma}(I[N]) .
\end{aligned}
$$

As was the case for $G$ above, a continuous modification $Q_{\mu \sigma}$ of $G_{\mu \sigma}$ can be obtained by defining

$$
Q_{\mu \sigma}(I[N]):=\int_{I[N]} \mathbf{1}_{C_{N}}(x) G_{\mu \sigma}(x, M, J) .
$$

Details of this argument are in [8], and in [9], section 6.

Changes of variable give an integrand like that of [7], page 52, Proposition 36 , so both $G_{\mu \sigma}$ and $Q_{\mu \sigma}$ are probabilities. In the Henstock integral approach to the analysis of Brownian motion and geometric Brownian motion, we use probability functions but not probability spaces. In other words, while we require appropriate probability functions for cylindrical intervals of the form 
$I[N]$ (which are, of course, measurable subsets of the sample space), the construction of the model does not need any consideration of the probabilities of subsets which are measurable in the classical Lebesgue, Borel or Baire senses.

This is a key point of the analysis, and, because of the focus on finitedimensional objects, it produces a considerable simplification in the representation and analysis of stochastic processes, and this follows through into the theory of derivative asset pricing.

The probabilities of outcomes which are arbitrary measurable subsets of the sample space are obtained as follows. If $A$ is a subset of the sample space and if $\int_{A} Q(I)$ exists, we take this to be $Q(A)$, the probability that $x \in A$. If $A$ is a measurable set in the sense of Kolmogorov-type probability measures $P$, then Theorem 4 of [8] implies that the probability $\int_{A} P(I)$ of $A$ (in the sense of Henstock) exists and the two are equal. This in turn implies that the pricing model we are developing is a generalization of the Lebesgue integral-based model.

$Q_{\mu \sigma}$ describes a geometric Brownian motion with growth rate $\mu$ and volatility $\sigma$, and $Q_{\mu \sigma}(I[N])$ gives the probability that a sample path $x$ of such a process satisfies $x(t) \in I[N]$ for $t \in N$.

Thus, where the classical Black-Scholes theory uses stochastic differential equations or Itô differentials [4] to specify and analyze stochastic processes, to achieve the same purpose we use the probability functions $Q_{\mu \sigma}$ defined on cylindrical intervals, or Henstock differentials, as we may call them.

\section{Risk-Neutral Valuation}

Suppose $r(t)$ is real for $\left.t \in] t_{0}, T\right]$. In financial theory, $r$ usually denotes the risk-free interest rate, and is non-negative. If a geometric Brownian motion is given by (2) above, with volatility $\sigma$ and growth rate $\mu$, we can construct probabilities on the sample space $C$ so that the resulting process has the same volatility $\sigma$ but a different growth rate $r$, so $x$ grows at the risk-free rate. The appropriate probabilities are $Q_{r \sigma}(x, N, I)$, so that expression (2) above has $\mu_{j}$ replaced by $r_{j}$. This is the change of measure that is required for risk-neutral valuation [2], and in this theory it is accomplished by using the elementary description of probability distributions.

Quite simply, using the Henstock integral model of stochastic processes, with cylindrical intervals replacing the measurable sets of the classical Itô model, we make the transition to the so-called risk-neutral world using an elementary argument involving the probability density function of a normal distribution, and we do not require the machinery of Itô calculus, Girsanov Theorem or Radon-Nikodym Theorem. 
All of the key points are covered at this stage, and the remainder of the discussion is given over to demonstrating the details of the Black-Scholes partial differential equation and pricing formula for a European call option.

Proposition 3. $g_{\mu \sigma}(x, N, I)$ is variationally equivalent to $G_{\mu \sigma}(x, N, I)$.

Proof. Follows from [7], page 54, Proposition 37, with the appropriate change of variable.

We now define a form of the kernel which is needed in the proof of the Black-Scholes partial differential equation (5) below. Let

$$
g^{*}(x(N)):=\prod_{j=2}^{n}\left[\exp \left\{-\frac{1}{2 \sigma_{j}^{2}} \frac{\left(\ln x_{j}-\ln x_{j-1}\right)^{2}}{t_{j}-t_{j-1}}\right\}\right]\left[2 \pi \sigma_{j}^{2}\left(t_{j}-t_{j-1}\right)\right]^{-\frac{1}{2}},
$$

which is like (1) above except that the product runs from $j=2$ instead of $j=1$, and let

$$
g^{*}(x(N), I(N)):=g^{*}(x(N)) \prod_{j=1}^{n} \frac{\left|I_{j}\right|}{x_{j}} .
$$

Following the pattern of notation that we have established, let

$$
G^{*}(I(N)):=\int_{I(N)} g^{*}(x(N), J(N)), G^{*}(I[N]):=G^{*}(I(N)),
$$

and $G^{*}(x, N, I):=G^{*}(I[N])$.

Proposition 4. If $\sigma(t)$ is bounded as $t \rightarrow t_{0}$, then $G(x, N, I)$ and $G^{*}(x, N, I)$ are variationally equivalent.

Proof.

$$
\left|G(x, N, I)-G^{*}(x, N, I)\right|=\int_{u_{1}}^{v_{1}} \int_{u_{2}}^{v_{2}} \cdots \int_{u_{n}}^{v_{n}}(a \times b) \frac{d x_{1}}{x_{1}} \frac{d x_{2}}{x_{2}} \cdots \frac{d x_{n}}{x_{n}}
$$

where

$$
a=\prod_{j=2}^{n}\left[\exp \left\{-\frac{1}{2 \sigma_{j}^{2}} \frac{\left(\ln x_{j}-\ln x_{j-1}\right)^{2}}{t_{j}-t_{j-1}}\right\}\right]\left[2 \pi \sigma_{j}^{2}\left(t_{j}-t_{j-1}\right)\right]^{-\frac{1}{2}}
$$

and

$$
b=\left|\left\{\exp \left(-\frac{1}{2 \sigma_{1}^{2}} \frac{\left(\ln x_{1}-\ln x_{0}\right)^{2}}{t_{1}-t_{0}}\right)\left(2 \pi \sigma_{1}^{2}\left(t_{1}-t_{0}\right)\right)^{-\frac{1}{2}}-1\right\}\right|
$$


With appropriate changes of variable, [7] Proposition 36 (page 52) and Proposition 45 (page 61) imply that a gauge $\gamma$ can be chosen so that, for any $\gamma$-fine division $\mathcal{E}_{\gamma}$ of $\mathbb{R}_{+}^{\left.t_{0}, T\right]}$,

$$
\begin{aligned}
& \left(\mathcal{E}_{\gamma}\right) \sum_{1}\left|G(x, N, I)-G^{*}(x, N, I)\right| \\
& <2 \int_{-\eta}^{\eta}\left|\left(\exp \left(-\frac{1}{2 \sigma_{1}^{2}} \frac{\left(y_{1}-y_{0}\right)^{2}}{t_{1}-t_{0}}\right)\right)\left(2 \pi \sigma_{1}^{2}\left(t_{1}-t_{0}\right)\right)^{-\frac{1}{2}}-1\right| d y_{1},
\end{aligned}
$$

where $\eta=\left(t_{1}-t_{0}\right)^{\alpha}$ with $\alpha<\frac{1}{2}$. This in turn is less than

$$
2 \times\left(2 \times\left(t_{1}-t_{0}\right)^{\alpha}\right) \times\left[\exp \left(-\frac{1}{2 \sigma_{1}^{2}}\left(t_{1}-t_{0}\right)^{2 \alpha-1}\right)\left(2 \pi \sigma_{1}^{2}\left(t_{1}-t_{0}\right)^{-\frac{1}{2}}\right)-1\right],
$$

and the term in square brackets is bounded as $t_{1} \rightarrow t_{0}$. Thus $\gamma$ can be chosen so that $\left(\mathcal{E}_{\gamma}\right) \sum\left|G(x, N, I)-G^{*}(x, N, I)\right|<\varepsilon$ for all divisions $\mathcal{E}_{\gamma}$, giving the result.

Proposition 5. If $\sigma(t)$ is bounded as $t \rightarrow t_{0}$, then $g(x, N, I)$ and $g^{*}(x, N, I)$ are variationally equivalent.

Proof. Follows from Propositions 1 and 4 above.

\section{Black-Scholes Theory}

The Black-Scholes model assumes that the underlying asset price process is geometric Brownian with growth rate $\mu(t)$, volatility $\sigma(t)$, and with sample paths $x$ in $C$. If the risk-free interest rate is given by $\left.r(t), t \in] t_{0}, T\right]$, a risk-free asset [2] such as a cash bond $b$, with initial value $b\left(t_{0}\right)$, will have a growth rate $r(t)$; so the value of the cash bond at time $t$ is $b(t)$ with $b(t)=b\left(t_{0}\right) e^{\int_{t_{0}}^{t} r(s) d s}$. Generally the growth rate $\mu(t)$ of risky assets is greater than $r(t)$, with the difference $\mu(t)-r(t)$ being known as the risk premium. We have already noted, in the previous section, how probability functions $Q_{r \sigma}$ can be constructed for the sample space so that, subject to these probability distributions, the asset process $x(t)$ has a mean growth rate of $r(t)$.

The discounted asset price process $y(t)$ is defined as

$$
y(t)=x(t) e^{-\int_{t_{0}}^{t} r(s) d s} .
$$

Given $N$ and $t \leq T\left(t>t_{0}\right)$, let $N^{\prime}$ denote $\left.\left.N \cap\right] t_{0}, t\right]$, let $I^{\prime}=I\left[N^{\prime}\right]$, let $x^{\prime}$ denote the projection of $x$ into $\overline{\mathbb{R}}_{+}^{\left.t_{0}, T\right]}$, and let $C^{\prime}$ denote the set of continuous $x$ in $\mathbb{R}_{+}^{t t, T]}$. Let $m=\max \left\{j: t_{j} \leq t, t_{j} \in N\right\}$, and let $R\left(N^{\prime}\right)$ denote

$$
\exp \left(\sum_{j=1}^{m} r\left(t_{j}\right)\left(t_{j}-t_{j-1}\right)\right) ;
$$


so $R\left(N^{\prime}\right)^{-1}=\exp \left(-\sum_{j=1}^{m} r\left(t_{j}\right)\left(t_{j}-t_{j-1}\right)\right)$.

If $G_{\mu \sigma}(x, N, I)$ is integrable in $\mathbb{R}_{+}^{\left.1 t_{0}, T\right]}$, then Fubini's Theorem ([7], page 37, Proposition 24) implies $G_{\mu \sigma}\left(x^{\prime}, N^{\prime}, I^{\prime}\right)$ is integrable in its domain.

For simplicity, omit the's in the following.

Proposition 6. If $r(t)$ is continuous, if $\sigma$ is constant and if $G_{\mu \sigma}$ is integrable in $\mathbb{R}_{+}^{\left[t_{0}, t\right]}$, then $e^{-\int_{t_{0}}^{t} r(s) d s} G_{\mu \sigma}(x, N, I)$ is variationally equivalent to $R(N)^{-1} G_{\mu \sigma}(x, N, I)$.

Proof. For each continuous $x$ choose $L(x)$ so that $N \supseteq L(x)$ implies

$$
\left|R(N)^{-1}-e^{-\int_{t_{0}}^{t} r(s) d s}\right|<\varepsilon \times\left(\int_{\mathbb{R}_{+}^{\left.j t_{0}, t\right]}} G_{\mu \sigma}\right)^{-1} .
$$

Thus $\gamma$ can be chosen so that, for all $\mathcal{E}_{\gamma}$,

$$
\left|\left(\mathcal{E}_{\gamma}\right) \sum e^{-\int_{t_{0}}^{t} r(s) d s} G_{\mu \sigma}-R(N)^{-1} G_{\mu \sigma}\right|<\varepsilon,
$$

giving the result.

Let $t_{0} \leq \tau_{1}<\tau_{2} \leq T$. A process $z(t)$ is a martingale relative to the probabilities $P$ (in other words $z(t)$ is a $P$-martingale) if $z\left(\tau_{1}\right)=\int_{C^{\prime}} z\left(\tau_{2}\right) P\left(I^{\prime}\right)$ for every choice of $\tau_{1}$ and $\tau_{2}$, where the ' now denotes projection into the dimensions $\left.] \tau_{1}, \tau_{2}\right]$.

Proposition 7. If $\sigma(t)$ is continuous, then $y(t)$ is a $Q_{r \sigma}$-martingale.

Proof. It is sufficient to show that $\int_{C^{\prime}} y(t) Q_{r \sigma}\left(I^{\prime}\right)=y\left(t_{0}\right)=x\left(t_{0}\right)$. Use the argument of [7], page 52, Proposition 36, along with the fact that $\int_{-\infty}^{\infty} u e^{-u^{2}} d u$ equals zero.

The following expressions are needed in the proof of the Black-Scholes partial differential equation below (5). Define $g_{\mu \sigma}^{*}(x(N))$ to be

$$
\prod_{j=2}^{n} \frac{\left[\exp \left\{-\frac{1}{2 \sigma_{j}^{2}}\left(\frac{\ln x_{j}-\ln x_{j-1}}{t_{j}-t_{j-1}}-\left(\mu_{j}-\frac{1}{2} \sigma_{j}^{2}\right)\right)^{2}\right\}\left(t_{j}-t_{j-1}\right)\right]}{\left[2 \pi \sigma_{j}^{2}\left(t_{j}-t_{j-1}\right)\right]^{\frac{1}{2}}}
$$

that is, expression (2) above with the product running from $j=2$ instead of $j=1$. Similarly, let $V^{*}(x, N ; \mu, \sigma)$ be expression (3) with the product running from $j=2$ instead of $j=1$. 
Let

$$
g_{\mu \sigma}^{*}(x(N), I(N)):=g_{\mu \sigma}^{*}(x(N)) \prod_{j=1}^{n} \frac{\left|I_{j}\right|}{x_{j}} .
$$

So $g_{\mu \sigma}^{*}(x(N), I(N))=g^{*}(x(N), I(N)) V^{*}$. Continuing with the same notational system we have used throughout, let

$$
g_{\mu \sigma}^{*}(x, N, I[N]):=g_{\mu \sigma}^{*}(x(N), I(N)) .
$$

Proposition 8. If $r(t)$ and $\sigma(t)$ are continuous, then $R^{*}(N)^{-1} g_{r \sigma}^{*}(x, N, I[N])$ and $R(N)^{-1} g_{r \sigma}(x, N, I[N])$ are variationally equivalent.

Proof. Follows from Propositions 5 and 6 above, and [7], page 54, Proposition 37.

As in Proposition 2 above, let $\left.\left.M=\left\{\tau_{1}, \ldots, \tau_{m}\right\} \subset\right] t_{0}, T\right]$ be fixed and let $h$ be a cylindrical functional satisfying $h(x)=h(x(M))$ for all $x$. Suppose $r$ is piecewise constant, with $r(t)=r\left(\tau_{j}\right)$ for $\left.\left.t \in\right] \tau_{l-1}, \tau_{j}\right], 1 \leq j \leq m$. Let $R(M)$ denote $\exp \left(\sum_{j=1}^{m} r\left(\tau_{j}\right)\left(\tau_{j}-\tau_{j-1}\right)\right)$.

Proposition 9. Under the conditions of Proposition 2 above,

$$
\int_{C} R(N)^{-1} h(x) G_{\mu \sigma}(x, N, I)=\int_{\mathbb{R}^{M}} R(M)^{-1} h\left(x(M) G_{\mu \sigma}(I(M)) .\right.
$$

Proof. Use the same argument as that used in Proposition 2.

This result implies that, in cases where the hypotheses of Proposition 9 hold, expectations relative to $Q$ can be obtained by finite-dimensional integrals.

The next result shows that, provided the functions involved are almost everywhere continuous, expectations relative to $Q$ can be obtained as limits of sequences of finite-dimensional integrals.

Let

$$
\tau_{j}^{(k)}:=t_{0}+\frac{j\left(T-t_{0}\right)}{k}, \text { for } 0 \leq j \leq k,
$$

so $\tau_{0}^{(k)}=t_{0}$ and $\tau_{k}^{(k)}=T$. Let $M^{(k)}$ denote $\tau_{1}^{(k)}, \ldots, \tau_{k}^{(k)}$. Let $\rho_{k}\left(x\left(M^{(k)}\right)\right):=$ $R\left(M^{(k)}\right)^{-1}$, and let

$$
\rho(x)=\left\{\begin{array}{lll}
\exp \left(-\int_{t_{0}}^{T} \mu(s) d s\right) & \text { if } & x \in C, \\
0 & \text { if } \quad x \notin C .
\end{array}\right.
$$

Let

$$
\Gamma_{k}\left(x\left(M^{(k)}, I\left(M^{(k)}\right)\right):=\rho_{k}\left(x\left(M^{(k)}\right)\right) G_{\mu \sigma}\left(x\left(M^{(k)}\right), I\left(M^{(k)}\right)\right) .\right.
$$


Proposition 10. If $r(s)$ is continuous for almost all $s$, then $\Gamma_{k}$ is integrable in $\mathbb{R}^{M^{(k)}}$ for each $k$, the sequence of integrals converges as $k \rightarrow \infty$, and the integral $\int_{C} \rho(x) Q_{\mu \sigma}(I)$ exists and equals $\lim _{k \rightarrow \infty} \int_{\mathbb{R}^{M^{(k)}}} \Gamma_{k}$.

Proof. Since $r(t)$ is non-negative, [7], page 67, Proposition 53 gives the result, using the Dominated Convergence Theorem.

The Arbitrage Theorem or Fundamental Theorem of Asset Pricing (see [3] Chapter 2) implies there exists a class $\mathcal{H}$ of functionals $h$ of $x \in C$ such that, if $P$ is a martingale probability for $x(t) \exp \left(-\int_{t_{0}}^{t} r(s) d s\right)$, then $P$ is also a martingale probability for $h(x) \exp \left(-\int_{t_{0}}^{t} r(s) d s\right)$. One such functional is $\max (x(T)-K, 0)$, which is the value at time $T$ of a European call option, ${ }^{1}$ on an asset with price process $x$, with exercise date $T$ and exercise price $K$.

Since this $h \in \mathcal{H}$ is a cylinder functional, with the dependence on $x$ determined by the values of $x$ in the single dimension $T$, if $r$ is constant, we can use Proposition 9, and, provided $\sigma$ is also assumed constant, a familiar evaluation of the integral gives the well known result [2] (page 91)

$$
x(t) \int_{-\infty}^{d_{1}} \frac{e^{-\frac{1}{2} u^{2}}}{\sqrt{2 \pi}} d u-K e^{-r(T-t)} \int_{-\infty}^{d_{2}} \frac{e^{-\frac{1}{2} u^{2}}}{\sqrt{2 \pi}} d u
$$

for the value at time $t$ of the European call option, where

$$
d_{1}=\frac{\ln \frac{x(t)}{K}+r+\frac{1}{2} \sigma^{2}(T-t)}{\sigma \sqrt{T-t}} \text { and } d_{2}=\frac{\ln \frac{x(t)}{K}+r-\frac{1}{2} \sigma^{2}(T-t)}{\sigma \sqrt{T-t}} .
$$

Using a conventional notation of finance, let $f$ denote the present value (time $t=t_{0}$ ) of a derivative asset whose dependence on the underlying asset price process $x$ is given by $h(x)$.

The foregoing argument can be summarized as follows.

Proposition 11. If $h \in \mathcal{H}$, then $f=\int_{C} h(x) \rho(x) Q_{r \sigma}(I)$ whenever the integral exists.

The following result gives a direct link between the risk-neutral expectation argument of Proposition 11 and the argument used by Black and Scholes in [1], in which a partial differential equation is solved.

Again taking $t=t_{0}$, let $\xi$ denote $x(t)$.

\footnotetext{
${ }^{1}$ An option, exercisable at some future time $T$, to buy a share for a price $K$, will only be exercised if the (unpredictable) value $x(T)$ of the share, at time $T$, is greater than $K$. Otherwise the option is not exercised and its value is 0 .
} 
Proposition 12. If $r(s)$ and $\sigma(s)$ are continuous from the right at $t$, and otherwise almost everywhere continuous, and if $f=\int_{C} h(x) \rho(x) Q_{r \sigma}(I)$ exists, then $f$ satisfies

$$
\frac{\partial f}{\partial t}+r(t) \xi \frac{\partial f}{\partial \xi}+\frac{1}{2} \sigma(t)^{2} \xi^{2} \frac{\partial^{2} f}{\partial \xi^{2}}=r(t) f
$$

Proof. By Proposition $3, \int_{\mathbb{R}_{+}^{\left.j t_{0}, T\right]}} R(N)^{-1} g_{r \sigma}(x, N, I)$ exists and equals $f$. Let $v(\xi, t)$ denote $R(N)^{-1} g_{r \sigma}(x, N, I)$. Then $v(\xi, t)=w(\xi, t) R^{*}(N)^{-1} g_{r \sigma}^{*}(x, N, I)$ where $w(\xi, t)$ is

$$
\frac{\exp \left[-2 \sigma\left(t_{1}\right)^{2}\left\{\frac{\ln x_{1}-\ln \xi}{t_{1}-t}-\left(r\left(t_{1}\right)-\frac{1}{2} \sigma\left(t_{1}\right)^{2}\right)\right\}^{2}\left(t_{1}-t\right)-r\left(t_{1}\right)\left(t_{1}-t\right)\right]}{2 \pi \sigma\left(t_{1}\right)^{2}\left(t_{1}-t\right)^{\frac{1}{2}}} .
$$

Note that $R^{*}$ and $g_{V}^{*}$ are independent of $\xi$ and $t$. By differentiation we find that $w$ satisfies

$$
\frac{\partial w}{\partial t}+r\left(t_{1}\right) \xi \frac{\partial w}{\partial \xi}+\frac{1}{2} \sigma\left(t_{1}\right)^{2} \xi^{2} \frac{\partial^{2} w}{\partial \xi^{2}}=r\left(t_{1}\right)
$$

and multiplying both sides by $R^{*}(N)^{-1} g_{r \sigma}^{*}(x, N, I)$ we get

$$
\frac{\partial v}{\partial t}+r\left(t_{1}\right) \xi \frac{\partial v}{\partial \xi}+\frac{1}{2} \sigma\left(t_{1}\right)^{2} \xi^{2} \frac{\partial^{2} v}{\partial \xi^{2}}=r\left(t_{1}\right) R^{*}(N)^{-1} g_{r \sigma}^{*}(x, N, I) .
$$

By Proposition 8 the right hand side of this equation is integrable, with integral $r f$. By the Dominated Convergence Theorem, when we integrate the left hand side term by term, the operations of integration and differentiation can be interchanged, giving

$$
\frac{\partial f}{\partial t}+r(t) \xi \frac{\partial f}{\partial \xi}+\frac{1}{2} \sigma(t)^{2} \xi^{2} \frac{\partial^{2} f}{\partial \xi^{2}}=r(t) f
$$

(Note the remarks on an analogous theorem concerning Schrödinger's equation in $[9]$.)

\section{References}

[1] F. Black and M. Scholes, The pricing of options and corporate liabilities, Journal of Political Economy, 81 (1973) 637-659.

[2] M. Baxter and A. Rennie, Financial Calculus: an introduction to derivative pricing, Cambridge, 1996. 
[3] J. E. Ingersoll, Theory of Financial Decision Making, Rowman and Littlefield, Savage, 1987.

[4] I. Karatzas and S. E. Shreve, Brownian Motion and Stochastic Calculus, Springer-Verlag, 1988.

[5] I. Karatzas and S. E. Shreve, Methods of Mathematical Finance, Springer, 1998.

[6] Y. K. Kwok, Mathematical Methods of Financial Derivatives, Springer, 1998.

[7] P. Muldowney, A General Theory of Integration in Function Spaces, Pitman Research Notes in Mathematics no. 153, Harlow, 1988.

[8] P. Muldowney, Topics in probability using generalised Riemann integration, Mathematical Proceedings of the Royal Irish Academy, 99B(1), 1999, 39-50.

[9] P. Muldowney, Feynman's path integrals and Henstock's non-absolute integration, Journal of Applied Analysis, 6(1), (2000), 1-24. 
P. Muldowney 